\title{
Multiple primary malignancies and subtle mucocutaneous lesions associated with a novel PTEN gene mutation in a patient with Cowden syndrome: Case report
}

\author{
Peter Vasovčák ${ }^{1 *}$, Mária Šenkeř́iková ${ }^{2}$ Jana Hatlováa ${ }^{3}$ Anna Křepelová ${ }^{1}$
}

\begin{abstract}
Background: Cowden syndrome (CS) is a cancer predisposition syndrome associated with increased risk of breast, thyroid, and endometrial cancers, and is characterized by development of benign mucocutaneous lesions.

Case presentation: Here we report on a 58-year-old woman with multiple primary malignancies and subtle mucocutaneous lesions such as small polyps and wart-like papulas. Over a period of 23 years, she developed various malignant neoplasms including thyroid, ovarian, stomach, and colon carcinomas, and a benign meningioma. Direct sequencing analysis of the PTEN gene revealed a novel germline mutation (c.438delT, p.Leu146X).
\end{abstract}

Conclusion: This case demonstrates that Cowden syndrome is a multi-system disease that can result in the development of multiple malignant and benign tumors.

\section{Background}

Cowden syndrome (CS, OMIM 158350) is an autosomal dominant disorder characterized by multiple hamartomas, which develop in the skin, thyroid gland, breast, gastrointestinal tract, and brain [1]. Germline mutations in the PTEN (phosphatase and tensin homolog deleted on chromosome ten) gene have been found in $80 \%$ of patients with CS [2]. The PTEN gene encodes a dualspecificity protein and lipid phosphatase that regulates the phosphoinositol-3-kinase/Akt signal pathway which can result in cell cycle arrest in the G1-phase and apoptosis [3]. PTEN can directly or indirectly dephosphorylate focal adhesion kinase (FAK), resulting in inhibition of cell migration and cell spreading [3-5].

Diagnosing CS remains a challenge due to the variations in its clinical presentation. A recent moleculargenetic study estimated that the incidence of CS was $1 / 200000$, although the actual incidence is likely to be higher [6]. The pathognomonic features of CS include mucocutaneous wart-like lesions, which are present in

\footnotetext{
* Correspondence: pevas78@hotmail.com

'Department of Biology and Medical Genetics, Charles University 2nd Faculty of Medicine and University Hospital Motol, Prague, Czech Republic Full list of author information is available at the end of the article
}

99\% of affected individuals before the age of 30 years [3]. The other most commonly reported manifestations are breast carcinomas, thyroid abnormalities, macrocephaly, hamartomatous polyps and mental retardation $[7,8]$. In women with CS, the lifetime risk of breast cancer is estimated to be $25-50 \%$, compared to the general female population risk of approximately $11 \%$. The lifetime risk of epithelial thyroid cancer can be as high as $10 \%$ in both males and females with CS. Follicular carcinoma is the most common CS-associated thyroid cancer, although papillary carcinomas have also been observed $[9,10]$.

The present report describes the identification of a novel PTEN germline mutation in a female CS patient who developed multiple primary tumors and subtle skin lesions.

\section{Case presentation}

A 55-year-old woman suspected to have CS was referred to the Department of Biology and Medical Genetics, University Hospital Motol, Prague, for PTEN gene analysis to confirm the diagnosis.

Her medical history was unremarkable until she developed a goiter at 34 years of age. At 45 years of age, she 
underwent a strumectomy. A histopathological examination confirmed a macrofollicular adenoma in the left thyroid lobe and an encapsulated thyroid tumor in the right thyroid lobe. At 54 years of age she underwent a total thyroidectomy due to a follicular carcinoma (Figure 1a).

At 43 years of age she underwent a hysterectomy for myomatosis and adnexectomy due to an adenopapilocarcinoma of the left ovary.

At the age of 49 years, the patient was admitted to hospital with epileptic paroxysms. A CT scan of the brain showed a left frontal lesion, which was the cause of the paroxysms. The lesion was surgically removed and histopathologically classified as a benign meningioma (Figure 1b). At 53 and 57 years of age, she was again surgically treated for meningioma recurrence and a steadily deteriorating condition. Despite those surgical interventions, there was a progressive decline in cognitive functions and memory.

The initial brain tumor finding together with the observed cachexia led to the suspicion of metastasis from an unknown primary cancer. Careful endoscopic examination revealed hundreds of polyps in the stomach, duodenum and colon. There were numerous mostly hyperplastic and hamartomatous polyps up to $10 \mathrm{~mm}$ in diameter in the stomach. Smaller and multiple sessile polyps were found in the duodenum. The esophagus was free of any polyps. Along the entire length of the colon were multiple histopathologically confirmed hyperplastic and inflammatory polyps as well as tubulovillous or villous adenomas of 4-10 $\mathrm{mm}$ in diameter, with low-grade dysplasia.

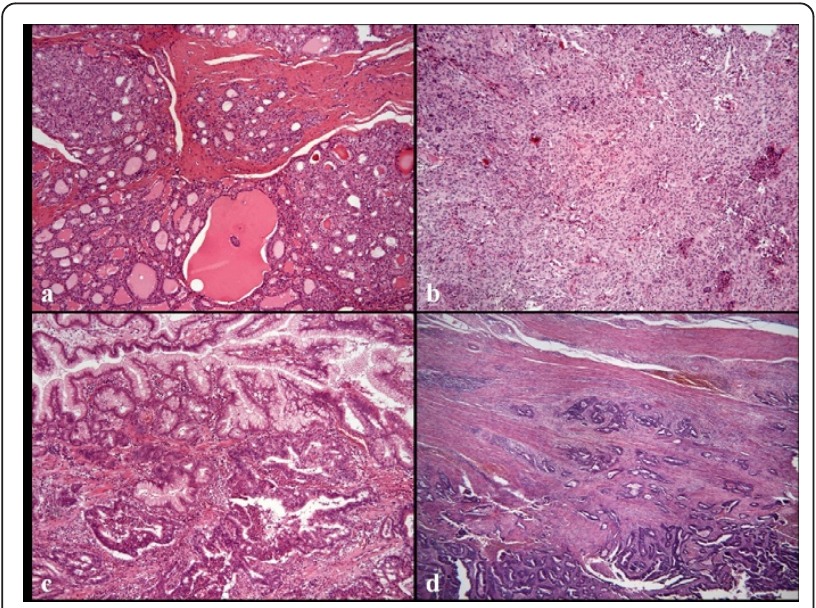

Figure 1 Histopathologic examination of the CS patient. a) Minimally invasive follicular carcinoma of the thyroid gland $(H \& E \times 100)$. b) Meningotheliomatous meningioma $(H \& E \times 100)$. c) Gastric hyperplastic polyp with adenocarcinoma (H\&E ×200). d) Tubular adenocarcinoma of the large bowel (H\&E $\times 100)$.
At the age of 55 years, the patient was examined by a clinical geneticist (MS) who observed macrocephaly, a fissured tongue and a polyp of $0.5 \mathrm{~cm}$ in diameter in the dorsal part of the oral cavity. A few wart-like papules on the forearms and chest were classified as senile verrucoid lesions. No other mucocutaneous lesions were found on the face or oral mucosa. The patient's mother, whose medical history was unknown to us, died of colon cancer at the age of 56 years. No other members of the family have had signs of CS or any cancer. The patient had one child who was negatively tested for mutations in the PTEN gene.

Several biopsies were performed throughout the gastrointestinal (GI) tract over a 9-year period, and none showed evidence of malignant lesions. Then at 57 years of age, histopathological examination of biopsy material from a hyperplastic polyp in the stomach revealed a well-differentiated intramucosal adenocarcinoma of $8 \mathrm{~mm}$ in diameter (Figure 1c). One year after the gastric cancer had been detected, the patient developed an aggressively growing synchronous adenocarcinoma of the anorectum (T3NOMO) and the sigmoid colon (T3N0M0) (Figure 1d). Abdominoperineal surgery (i.e., the Miles' operation) was performed. However, the patient died at post-operative 1 month due to unmanageable complications.

\section{Methods}

This study was approved by the Ethics Committee of the University Hospital Motol, Prague, according to the Helsinki Principles. After receiving written informed consent, the genomic DNA of the patient was isolated from blood leukocytes using a Genomic DNA Purification Kit (Gentra Systems, Minneapolis, MI, USA) according to the manufacturer's guide. The genomic DNA was amplified using intronic primers (sequences are available upon request) flanking the nine exons and a promoter region of the PTEN gene [11]. The PCR products were purified using a SureClean PCR purification kit (Bioline, London, UK). Bidirectional cycle sequencing of the PCR products was performed using a BigDye Terminator v3.1 Cycle Sequencing kit and an ABI 3130 Genetic Analyzer (both from Applied Biosystems, Foster City, CA, USA).

\section{Results and Discussion}

Examination of the genomic DNA revealed a novel heterozygous mutation, c.438delT, in exon 5 (Figure 2). That mutation is predicted to lead to a frameshift that results in formation of a premature stop codon (p.Leu146X) for PTEN protein translation. The mutation is considered to be pathogenic and causative for CS disease. 


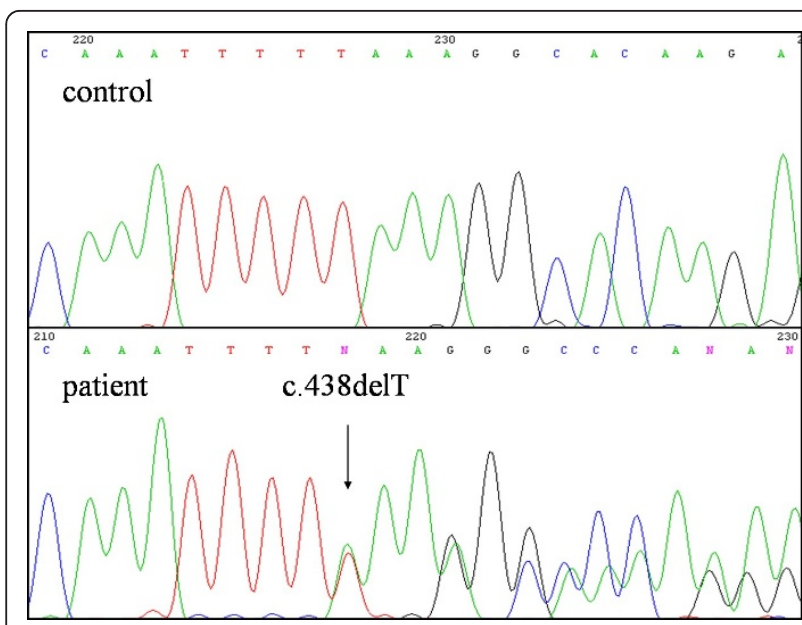

Figure 2 DNA sequencing electropherograms. Upper panel: Electropherogram of the PTEN gene, exon 5, from a healthy control. Lower panel: Electropherogram of the PTEN gene, exon 5, from the Cowden syndrome patient. Note the heterozygous deletion mutation at c.438delT (arrow).

More than $80 \%$ of patients who strictly meet the International Cowden Consortium criteria are found to harbor a PTEN mutation [12]. Mucocutaneous lesions are present in $99 \%$ of CS patients before the age of 30 years [3]. However, the present patient was not reported to have had mucocutaneous lesions until 55 years of age, when a targeted examination revealed subtle skin lesions. The only visible wart-like papules were found on her forearms. Such lesions are commonly found in the general population and could therefore have been overlooked or considered unremarkable.

The most common malignancies associated with CS are breast and thyroid carcinomas. Benign breast lesions (fibrocystic breast disease, fibroadenomas) and breast adenocarcinomas develop in about $76 \%$ and $25-50 \%$ of CS-affected females, respectively [3]. The average age of a breast cancer diagnosis in CS patients is between 38 and 46 years [13]. Marsh et al. observed an association between the presence of a PTEN mutation and malignant breast disease [12]. In our patient, a mammographic examination at 57 years of age showed no evidence of any breast lesions.

Benign thyroid lesions occur in as many as $60 \%$ and thyroid cancers in up to $10 \%$ of CS patients [3,9]. Our patient developed a follicular adenocarcinoma at 54 years of age, despite regular follow-up and treatment since her first admission to hospital due to a goiter at 34 years old.

Gynecological abnormalities are reported in $44 \%$ of females with CS [7]. Endometrial cancer is a major diagnostic criterion for CS, with an estimated frequency of 5-10\% [7]. Ovarian tumors are rare in CS, with only one report documenting an ovarian dysgerminoma in a CS patient [14]. The hypothesis that a germline mutation of the PTEN gene was implicated in the formation of ovarian tumors in CS patients was supported by the identification of a somatic loss of heterozygosity $(\mathrm{LOH})$ in the wild-type allele [14]. The present patient had myomatosis of the uterus and adenocarcinoma of the left ovary at the age of 43 years. Unfortunately, ovary tumor tissue was not available for $\mathrm{LOH}$ analysis.

Macrocephaly and Lhermitte-Duclos disease (dysplastic cerebellar gangliocytoma) are also major pathognomonic diagnostic criteria of CS [15]. Meningiomas (benign brain tumors) have also been described in CS patients, albeit rarely [16-19]. In our patient, the discovery of a meningioma launched an intensive and thorough follow-up which revealed additional and continually appearing symptoms. Benign brain lesions accompanied by epileptic paroxysms and a steadily deteriorating clinical condition were her main health problems.

Gastrointestinal polyps (mostly classified as hamartomatous, hyperplastic, inflammatory, juvenile, lymphomatous and adenomatous) are commonly reported in CS patients, with frequencies ranging between $35-85 \%$ and higher than $90 \%$ in Western and Japanese patients, respectively $[9,20-24]$. That difference in frequency is considered to reflect that GI examinations are more common in Japan [24]. In a study involving 126 CS patients, Marra et al., reported that of the 42 patients who underwent a complete GI examination, 36 (85\%) had GI polyps. Non-adenomatous polyps that are generally asymptomatic may be more common in CS patients, especially when GI investigations are not part of the recommended surveillance for CS. Although an association between CS and GI cancer remains a topic of debate, reports describing malignant GI tumors in CS patients have been published [9,21-23, 25-27]. Although non-adenomatous polyps have a weak or absent malignant potential, there is evidence that carcinomas may arise within non-adenomatous polyps $[26,28$, and the present study]. Marra et al. stated that adenomatous polyps represent approximately $25 \%$ of CS polyps, and have a higher malignant potential than non-adenomatous polyps [22]. During a 9-year follow-up period, the present patient developed numerous GI polyps with various histopathological features (hyperplastic, inflammatory, hamartomatous and adenomatous). Ultimately, three polyps (a hyperplastic polyp in the stomach, and adenomatous polyps in the rectum and the sigmoid colon) progressed to macroscopic carcinomas.

\section{Conclusion}

Although breast and thyroid cancers are the predominant malignancies in CS patients, it should be emphasized that benign and/or malignant tumors may also develop in the GI tract from pre-existing polyps, and in the genitourinary tract or in the brain. Most of the studies that have examined cancer in CS patients do not 
report the lifetime risk of development of a malignant neoplasm because the patients are relatively young. We propose that meningiomas and GI tract cancers, albeit rare, should be a component of the definition of CS. Physicians who might encounter CS patients should be aware of the possible neurological and/or GI tract manifestations.

\section{Consent}

Written informed consent was obtained from the patient for publication of this case report and for the use of the accompanying images. A copy of the written consent can be obtained from the Editor-in-Chief of this journal.

\section{Acknowledgements}

We thank Evzen Krepela, MD PhD and Petra Hedvičáková, for critical reading and editing of the manuscript.

Supported by: VZ MZO 00064203

\section{Author details}

'Department of Biology and Medical Genetics, Charles University 2nd Faculty of Medicine and University Hospital Motol, Prague, Czech Republic. ${ }^{2}$ Department of Medical Genetics, University Hospital, Hradec Králové, Czech Republic. ${ }^{3}$ Fingerland's Department of Pathology, Faculty of Medicine, Hradec Králové, Czech Republic.

\section{Authors' contributions}

PV carried out the molecular genetic study including the DNA sequencing, and drafted the manuscript. MS identified and diagnosed the patient. JH prepared, read and classified the histological samples. AK designed the study and revised the manuscript. All authors read and approved the final manuscript.

\section{Competing interests}

The authors declare that they have no competing interests.

Received: 7 August 2010 Accepted: 15 March 2011

Published: 15 March 2011

\section{References}

1. Lloyd KM, Dennis M: Cowden's disease. A possible new symptom complex with multiple system involvement. Ann Intern Med 1963, 58:136-142.

2. Liaw D, Marsh DJ, Li J, Dahia PL, Wang SI, Zheng Z, Bose S, Call KM, Tsou HC, Peacocke M, et al: Germline mutations of the PTEN gene in Cowden disease, an inherited breast and thyroid cancer syndrome. Nat Genet 1997, 16:64-67.

3. Eng C: PTEN: one gene, many syndromes. Hum Mutat 2003, 22:183-198.

4. Tamura M, Gu J, Matsumoto K, Aota S, Parsons R, Yamada KM: Inhibition of cell migration, spreading, and focal adhesions by tumor suppressor PTEN. Science 1998, 280:1614-1617.

5. Tamura M, Gu J, Danen EH, Takino T, Miyamoto S, Yamada KM: PTEN interactions with focal adhesion kinase and suppression of the extracellular matrix-dependent phosphatidylinositol 3-kinase/Akt cell survival pathway. J Biol Chem 1999, 274:20693-20703.

6. Nelen MR, van Staveren WC, Peeters EA, Hassel MB, Gorlin RJ, Hamm H, Lindboe CF, Fryns JP, Sijmons RH, Woods DG, et al: Germline mutations in the PTEN/MMAC1 gene in patients with Cowden disease. Hum Mol Genet 1997, 6:1383-1387.

7. Eng C: Will the real Cowden syndrome please stand up: revised diagnostic criteria. J Med Genet 2000, 37:828-830.

8. Nelen MR, Padberg GW, Peeters EA, Lin AY, van den Helm B, Frants RR, Coulon V, Goldstein AM, van Reen MM, Easton DF, et al: Localization of the gene for Cowden disease to chromosome 10q22-23. Nat Genet 1996, 13:114-116.
9. Starink TM, van der Veen JP, Arwert F, de Waal LP, de Lange GG, Gille JJ, Eriksson AW: The Cowden syndrome: a clinical and genetic study in 21 patients. Clin Genet 1986, 29:222-233.

10. Waite KA, Eng C: Protean PTEN: form and function. Am J Hum Genet 2002, 70:829-844.

11. Zhou XP, Waite KA, Pilarski R, Hampel H, Fernandez MJ, Bos C, Dasouki M, Feldman GL, Greenberg LA, Ivanovich J, et al: Germline PTEN promoter mutations and deletions in Cowden/Bannayan-Riley-Ruvalcaba syndrome result in aberrant PTEN protein and dysregulation of the phosphoinositol-3-kinase/Akt pathway. Am J Hum Genet 2003, 73:404-411.

12. Marsh DJ, Coulon V, Lunetta KL, Rocca-Serra P, Dahia PL, Zheng Z, Liaw D, Caron S, Duboue B, Lin AY, et al: Mutation spectrum and genotypephenotype analyses in Cowden disease and Bannayan-Zonana syndrome, two hamartoma syndromes with germline PTEN mutation. Hum Mol Genet 1998, 7:507-515.

13. Pilarski R, Eng C: Will the real Cowden syndrome please stand up (again)? Expanding mutational and clinical spectra of the PTEN hamartoma tumour syndrome. J Med Genet 2004, 41:323-326.

14. Cho MY, Kim HS, Eng C, Kim DS, Kang SJ, Eom M, Yi SY, Bronner MP: First report of ovarian dysgerminoma in Cowden syndrome with germline PTEN mutation and PTEN-related 10q loss of tumor heterozygosity. Am J Surg Pathol 2008, 32:1258-1264.

15. Gustafson S, Zbuk KM, Scacheri C, Eng C: Cowden syndrome. Semin Oncol 2007, 34:428-434.

16. Lok C, Viseux V, Avril MF, Richard MA, Gondry-Jouet C, Deramond H, Desfossez-Tribout C, Courtade S, Delaunay M, Piette F, et al: Brain magnetic resonance imaging in patients with Cowden syndrome. Medicine (Baltimore) 2005, 84:129-136.

17. Lyons $\mathrm{CJ}$, Wilson $\mathrm{CB}$, Horton JC: Association between meningioma and Cowden's disease. Neurology 1993, 43:1436-1437.

18. Rademaker J, Kim YJ, Leibecke T, Raman SS, Voit C: Cowden disease: CT findings in three patients. Abdom Imaging 2005, 30:204-207.

19. Rimbau J, Isamat F: Dysplastic gangliocytoma of the cerebellum (Lhermitte-Duclos disease) and its relation to the multiple hamartoma syndrome (Cowden disease). J Neurooncol 1994, 18:191-197.

20. Chen YM, Ott DJ, Wu WC, Gelfand DW: Cowden's disease: a case report and literature review. Gastrointest Radiol 1987, 12:325-329.

21. Chilovi F, Zancanella L, Perino F, Wallnoefer W, Vigl EE, Colombetti V, Dobrilla G: Cowden's disease with gastrointestinal polyposis. Gastrointest Endosc 1990, 36:323-324.

22. Marra G, Armelao F, Vecchio FM, Percesepe A, Anti M: Cowden's disease with extensive gastrointestinal polyposis. J Clin Gastroenterol 1994, 18:42-47.

23. Salem OS, Steck WD: Cowden's disease (multiple hamartoma and neoplasia syndrome). A case report and review of the English literature. J Am Acad Dermatol 1983, 8:686-696.

24. Sawada T, Hamano N, Satoh H, Okada T, Takeda Y, Mabuchi H: Mutation analysis of the PTEN/MMAC1 gene in Japanese patients with Cowden disease. Jpn J Cancer Res 2000, 91:700-705.

25. Gorensek M, Matko I, Skralovnik A, Rode M, Satler J, Jutersek A: Disseminated hereditary gastrointestinal polyposis with orocutaneous hamartomatosis (Cowden's disease). Endoscopy 1984, 16:59-63.

26. Hamby LS, Lee EY, Schwartz RW: Parathyroid adenoma and gastric carcinoma as manifestations of Cowden's disease. Surgery 1995, 118:115-117.

27. Taylor AJ, Dodds WJ, Stewart ET: Alimentary tract lesions in Cowden's disease. Br J Radiol 1989, 62:890-892.

28. Bosserhoff AK, Grussendorf-Conen El, Rubben A, Rudnik-Schoneborn S, Zerres K, Buettner R, Merkelbach-Bruse S: Multiple colon carcinomas in a patient with Cowden syndrome. Int J Mol Med 2006, 18:643-647.

\section{Pre-publication history}

The pre-publication history for this paper can be accessed here: http://www.biomedcentral.com/1471-2350/12/38/prepub

doi:10.1186/1471-2350-12-38

Cite this article as: Vasovčák et al:: Multiple primary malignancies and subtle mucocutaneous lesions associated with a novel PTEN gene mutation in a patient with Cowden syndrome: Case report. BMC Medical Genetics 2011 12:38. 